\title{
Application of Big Data Deep Learning in Auxiliary Diagnosis of Lower Extremity Arteriosclerosis Obliterans
}

\author{
Linbo Liu1*, Yang Liu², Hongjun Wang33, Yi Zhang1, Zhijie Liao', Shengdong Du ${ }^{3}$ \\ ${ }^{1}$ The Third Hospital of Mianyang, Sichuan Mental Health Center, Mianyang 621000, Sichuan Province, China \\ ${ }^{2}$ Affiliated Hospital of Chengdu University of Traditional Chinese Medicine, Chengdu 610072, Sichuan Province, China \\ ${ }^{3}$ School of Computing and Artificial Intelligence, Southwest Jiaotong University, Chengdu 611756, Sichuan Province, China \\ *Corresponding author: Linbo Liu, 22646275@qq.com

\begin{abstract}
At present, the incidence rate of arteriosclerosis obliterans (LEASO) of the lower extremities is significantly increased by aging and lifestyle changes. It is of great importance to predict the LEASO effectively and accurately by analyzing the imaging data of the lower extremities ${ }^{[1]}$. At this stage, China has entered the era of big data and artificial intelligence. Medical institutions at all levels can produce a large number of lower limb vascular image data every day. Using big data deep learning technology to intelligently analyze a large number of image data, and then carry out auxiliary diagnosis, so as to improve the diagnosis and treatment effect of LEASO is the focus of clinical research.
\end{abstract}

Keywords: Big data; Deep learning; Arteriosclerosis obliterans of lower limbs; Auxiliary diagnosis

Publication date: September 2021; Online publication: September 30, 2021

\section{Introduction}

LEASO is a chronic progressive ischemic disease of the lower limbs due to the formation of atherosclerotic plaques of the lower extremities. The clinical incidence rate is increasing significantly year by year. At this stage, the research on LEASO mainly focuses on the selection of surgical scheme and postoperative followup evaluation of patients requiring surgical treatment, and there is still a lack of research and Analysis on the early diagnosis and prediction methods of LEASO ${ }^{[2-3]}$. In order to avoid the adverse effects of doctors' lack of clinical experience and insufficient resources of medical institutions on LEASO diagnosis, this paper focuses on the application of big data processing, artificial intelligence and deep learning technology in LEASO diagnosis, hoping to provide reference for clinical diagnosis and treatment of LEASO.

\section{Analysis on the current situation of LEASO diagnosis methods}

LEASO is a multifactorial atherosclerotic plaque in the lower limbs, which can lead to lower limb artery stenosis or occlusion. The patient's condition is characterized by chronic progressive progress. At present, the incidence rate of LEASO is significantly increased due to the aging of population and lifestyle changes. Statistics show that the overall incidence rate of LEASO in China is about 3-10\%, among which the incidence rate of $15-20 \%$ is over 75 years old. The patients' condition is progressively aggravated. Early diagnosis and timely intervention can significantly improve the prognosis of ${ }^{[4-6]}$.

The conventional methods for clinical diagnosis of LEASO are color Doppler ultrasound and CTA. Color Doppler ultrasonography can clearly display the morphological structure of vascular wall and vascular cavity, and determine hemodynamic indexes. It is noninvasive and easy to operate. It can 
accurately diagnose arteriosclerosis stenosis, and its sensitivity and specificity are more than $90 \%$. However, ultrasonography has high requirements for doctors' clinical experience and is prone to misdiagnosis and missed diagnosis. It is often used as the main scheme for large-scale screening and postoperative followup ${ }^{[7-8]}$. CTA examination can show the location, degree and scope of arterial tissue lesions, with high diagnostic accuracy, but the diagnostic cost is expensive, special equipment is required, and contrast agents are required during the diagnosis, which can cause varying degrees of damage to the patient's renal function ${ }^{[9-10]}$. Some medical institutions take DSA examination as the gold standard for the diagnosis of LEASO. This scheme can clearly display the scope, degree and location of the patient's lesions, evaluate the collateral circulation and distal outflow tract, and complete the internal treatment of vascular lumen. However, this examination method is invasive and can lead to the aggravation of limb ischemia ${ }^{[11-12]}$. At present, the demand of Chinese residents for medical services has increased significantly, the imbalance of medical resources between urban and rural areas is prominent, the equipment allocation of some grass-roots medical institutions is incomplete, and the talent reserve is insufficient, resulting in the low early diagnosis rate of chronic diseases such as LEASO, which seriously affects the effect of disease treatment ${ }^{[13]}$. Big data processing technology can integrate and make use of the technical advantages and experience of class III medical institutions in diagnosing LEASO to realize the intelligent diagnosis of LEASO, which helps to improve the diagnosis and treatment level of grass-roots medical institutions and avoid doctors' influencing the disease diagnosis and treatment results due to factors such as insufficient clinical experience and equipment. Its clinical application value is worthy of in-depth research.

\section{Analysis on core problems and technical route of image big data intelligent diagnosis LEASO}

The main problems to be solved in image big data intelligent diagnosis of LEASO include how to realize quantitative diagnosis and evaluation of LEASO by using LESO image video sequence lesion tracking and automatic detection technology; How to improve the single data-driven mode to form a LEASO diagnosis mode combining image expert knowledge, clinical detection and image ${ }^{[14]}$. The technical route of image big data intelligent diagnosis LEASO is relatively complex. It is necessary to give priority to the establishment of image big data intelligent diagnosis LEASO intelligent image database, which includes patient medical records, clinical indicators, images, expert knowledge, etc. After that, it is necessary to preprocess the database, establish a perfect image database, and form a multi-scale, cross time, feature and semantic data fusion through feature association, representation and extraction. After the above steps are completed, focus location and tracking (semi supervised deep learning model, multi-scale adaptive tracking strategy, deep learning and f-rcnn), feature extraction and fusion (deep enhanced feature fusion learning, risk diagnosis quantitative evaluation algorithm, feature modeling and small sample learning) ${ }^{[15]}$.

\section{Image big data intelligent diagnosis and LEASO prediction analysis based on convolutional support vector machine}

\subsection{Convolutional neural network and support vector machine}

Convolution neural network is a representative algorithm of deep learning. It is a pre feedback neural network based on neural network and adding convolution operation, mainly including feature extraction and hierarchical classification. Feature extraction requires low dimensional data to effectively represent the relevant content of the image. The classification involves complex contents. Researchers detect and classify pulmonary tuberculosis in CT, and detect and classify skin cancer by using human skin tissue photos. According to the characteristics of medical images, the researchers optimized the computational cost of google net model and introduced the basic mode of model fusion. The results show that the image classification effect of the fusion mode is significantly better than that of the original model. Support vector machine is a linear classifier. Researchers use support vector machine to comprehensively analyze renal 
ultrasound images, which can realize the accurate diagnosis of a variety of renal diseases. The accuracy of routine ECG in the diagnosis of acute myocardial infarction is low. The comprehensive analysis of gene expression related data by support vector machine can significantly improve the diagnostic accuracy ${ }^{[16]}$.

\subsection{Construction of convolutional support vector machine model}

\subsubsection{Convolutional support vector machine}

In the process of constructing the mode, it is necessary to give priority to establishing a complete convolution neural network framework structure including full connection layer, pooling layer and convolution layer. There are certain differences in the output patterns of different levels. The convolution layer contains several convolution cores, which can extract the detailed characteristics of the output data. The specific calculation formula of the input and output of the convolution layer is

$$
\begin{aligned}
& \text { (1) } z_{n, v}=\sum_{i=-\infty}^{\infty} \sum_{j=-\infty}^{\infty} x_{i+n j+1}^{l-1} \cdot K_{\text {roti } j}^{l}-\not(i, j)+b^{l} \text {, } \\
& \text { (2) } \quad x(i, j)=\left\{\begin{array}{l}
1,0 \leq i, j \leq n \\
0, \text { others }
\end{array}\right\} \text {, } \\
& \text { (3) } a_{u_{n} v}^{l}=f\left(z_{u_{w},}^{l}\right) \text {, }
\end{aligned}
$$

The output characteristics of the upper layer are input as the pool layer, which can complete the law and screening of data information. The specific calculation formula is

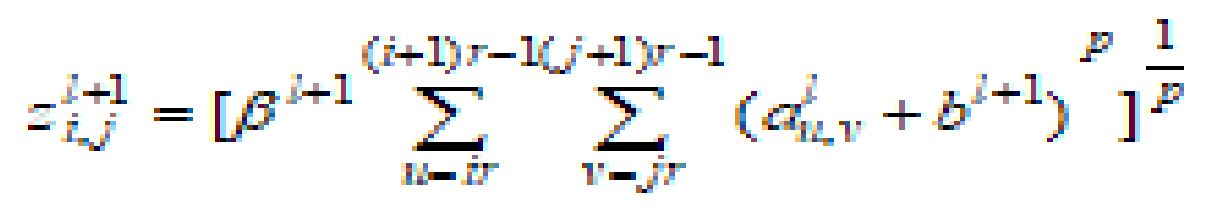

Through the analysis of the formula, the position relationship between the hyperplane and the sample can be determined, and then the accurate prediction category of the sample can be obtained.

\subsubsection{Comparative algorithm analysis}

The comparison algorithms mainly include naive Bayes, support vector machine, convolutional neural network, etc. naive Bayes belongs to the classification algorithm. The probability statistics theory is used to classify the data. The precondition is that the data attributes are independent. The maximum output of probability can be realized through this algorithm. Support vector machine belongs to linear classification mode and has good generalization ability. It can realize accurate classification by inputting the classification value of specific label in operation. Convolution neural network belongs to pre feedback neural network. Convolution related operations are integrated in the solution process, and it has a deep structure. This model has good application effects in natural language processing and computer vision.

\subsubsection{Algorithm related description}

The CT images of patients with LEASO were included as the research data office resources. The structure of single-layer pooling, three-layer convolution and single-layer full connection layer was adopted, and SVM classifier was introduced. The number of convolution kernels used in 1 convolution layer is $6(5 \times 5)$, 
the number of convolution kernels used in the 2-layer convolution layer is $8(3 \times 3)$, the number of convolution kernels used in the 3-layer convolution layer is $10(3 \times 3)$, rely is used as the activation function.

\subsubsection{Basic process analysis of LEASO prediction}

The data preparation of LEASO prediction needs to be completed first, followed by training data and test data. In the process of training data, training set and verification set can be formed, and support vector machine, naive Bayes, convolutional neural network and convolutional support vector machine can be extended to form verification model together with test data. In the data preparation stage, a suitable number of LEASO patient data should be included and divided into test data and training data. The test data is mostly used to evaluate the performance of the model, and the training data is used to train and improve the model. In the process of data preprocessing, the picture needs to be adjusted to $64 \times 64$. Reasonably set the gray image and input it into $\mathrm{SVB}, \mathrm{CNNs}$ and $\mathrm{Nb}$ for simulation training. During the test, the patient picture shall be used as the input data, and the input value shall be included in the training model, so as to achieve accurate prediction.

\section{Conclusion}

At present, the clinical incidence rate of LEASO is increasing year by year. It is important to accurately predict and diagnose the disease and implement targeted treatment intervention. Image big data intelligent diagnosis LEASO is based on the deep-seated intelligent extraction of vascular image features. It can realize the automatic detection and diagnosis of LEASO by using modeling and multimodal algorithm, convolution support vector machine model, etc. through the strong feature extraction and classification ability of neural network, it can realize the accurate prediction of LEASO, which is worthy of comprehensive promotion and application.

\section{Funding}

Scientific research project of Sichuan Provincial Health Commission "auxiliary diagnosis of lower extremity arteriosclerosis obliterans based on deep learning of big data," No.:18PJ488.

\section{Disclosure statement}

The author declares no conflict of interest.

\section{References}

[1] Zhang Y, et al., 2021, Application of Deep Learning in Laboratory Medicine Under the Background of Big Data. Electronic Components and Information Technology, 5(4): 201-202.

[2] Guo L, He H, Liu L., 2020, Application of Deep Learning in Medical Image Big Data. Network Security Technology and Application, (4): 131-132.

[3] Lu S, Liu L., 2015, Research Progress on Health Education of Lower Extremity Arteriosclerosis Obliterans. Chinese Journal of Arteriosclerosis, 23(06): 645-648.

[4] Chen Z, Yang V., 2018, Progress in Endovascular Treatment of Lower Extremity Arteriosclerosis Obliterans. Chinese Journal of Basic and Clinical Medicine, (1): 3-9.

[5] Vascular Surgery Group, 2015, Branch of Surgery, Chinese Medical Association. Guidelines for the Diagnosis and Treatment of Lower Extremity Arteriosclerosis Obliterans. Chinese Journal of Medicine, 95(24): 145-151. 
[6] Aboyans V, Sevestre MA, Desormais I, et al., 2018, Epidemiology of Lower Extremity Artery Disease. Presse Med, 47(1): 38-46.

[7] Xia X, Song Y, 2018, Application Value of Color Doppler Ultrasound Blood Flow Imaging in the Diagnosis of Lower Extremity Arteriosclerosis Obliterans. Journal of Practical Medical Imaging, 19(3): 202-205.

[8] Xia X, Song Y, 2018, Discussion on the application value of color Doppler ultrasound blood flow imaging in the diagnosis of lower extremity arteriosclerosis obliterans. Journal of practical medical imaging, (3): 202-205.

[9] Li L, Liu B, Li X, et al., 2020, Preliminary study on CT imaging analysis of muscle ischemia in lower extremity arteriosclerosis obliterans. Journal of Practical Radiology, (7): 1055-1058.

[10] Wang W, Wang X, Wang C, et al., 2020, Application value of CT angiography in the diagnosis of lower extremity arteriosclerosis obliterans. Chinese Journal of CT and MRI, 18(1): 149-152.

[11] Guo J, Guo L, Qi L, et al., 2018, Clinical study on surgical treatment of 4602 patients with chronic ischemia of lower limbs caused by single center lower extremity arteriosclerosis obliterans. Chinese Journal of Physicians, 20(12): 1787-1791.

[12] Gu J, Wang Y, Sun P, 2019, Efficacy analysis of endovascular interventional therapy for lower extremity arteriosclerosis obliterans. Journal of vascular and endovascular surgery, (2): 100-102, 155.

[13] Shi X, Zhao J, Wang L, et al., 2021, Overview of precision medical applications based on big data analysis technology. China hospital management, 41(5): 26-31.

[14] Lian W, Nie H, Yuan Y, et al., 2021, Clinical Significance of Endothelin-1 And C Reaction Protein in Restenosis After the Intervention of Lower Extremity Arteriosclerosis Obliterans. Journal of Investigative Surgery, 34(7).

[15] Chen Z, Chen X, de olivira JE, et al., 2019, Application of deep learning in equipment fault prediction and health management. Journal of instrumentation, 40(9): 206-226.

[16] Chen L, Zhang X, Jiang F, et al., 2021, Therapeutic Effects of Interventional Therapy and Conservative Therapy on Lower Extremity Arteriosclerosis Obliterans and Risk Factors for Prognosis. Artery Research, 27(2). 